\title{
Simulation of Geometrical Parameters of Screen Printed Electrode (SPE) for Electrochemical-Based Sensor
}

\author{
Nurul Amira Farhana Roslan \\ Department of Electrical and \\ Computer Engineering, \\ Kulliyyah of Engineering, \\ IIUM, Kuala Lumpur, Malaysia \\ nrlamirafarhana@gmail.com
}

\author{
Anis Nurashikin Nordin \\ Department of Electrical and \\ Computer Engineering, \\ Kulliyyah of Engineering, \\ IIUM, Kuala Lumpur, Malaysia \\ anisnn@iium.edu.my
}

\author{
Rosminazuin Ab Rahim \\ Department of Electrical and \\ Computer Engineering, \\ Kulliyyah of Engineering, \\ IIUM, Kuala Lumpur, Malaysia \\ rosmi@iium.edu.my
}

\begin{abstract}
Mohd Saiful Riza Bashri
Department of Science,

Kulliyyah of Engineering,

IIUM, Kuala Lumpur, Malaysia

mohdsaifulriza@iium.edu.my
\end{abstract}

Lim Lai Ming

Jabil Circuit Sdn. Bhd, Bayan

Lepas Industrial Park Phase 4,

Penang, Malaysia

laiming_lim@jabil.com

\author{
Aliza Aini Md Ralib \\ Department of Electrical and \\ Computer Engineering, \\ Kulliyyah of Engineering, \\ IIUM, Kuala Lumpur, Malaysia \\ alizaaini@iium.edu.my
}

Muhammad Irsyad Suhaimi

Jabil Circuit Sdn. Bhd, Bayan

Lepas Industrial Park Phase 4,

Penang, Malaysia

Muhammad_Irsyad@jabil.com

Gandi Sugandi

Research Center for Electronics and Telecommunications,

Bandung, Jawa Barat, Indonesia gandi511@yahoo.com

\author{
Nor Farahidah Za'bah \\ Department of Electrical and \\ Computer Engineering, \\ Kulliyyah of Engineering, \\ IIUM, Kuala Lumpur, Malaysia \\ adah510@iium.edu.my
}

\author{
Zambri Samsudin \\ Jabil Circuit Sdn. Bhd, Bayan \\ Lepas Industrial Park Phase 4, \\ Penang, Malaysia \\ zambrisamsudin@jabil.com
}

\begin{abstract}
Screen printing is a known method to produce disposable and low-cost sensors. Depending on the application such as food analysis, environmental health monitoring, disease detection and toxin detection, screen-printed electrodes can be fabricated in a variety of sizes and shapes. Modification of the electrode's material and geometrical dimension may be done to produce effective screen-printed three-electrodes system. Thus, the effects of varying the working electrode (WE) area in radius of $0.9 \mathrm{~mm}$ to $2 \mathrm{~mm}$, gap spacing between electrodes ranging from $0.5 \mathrm{~mm}$ to 1.6 $\mathrm{mm}$, and the width of the counter electrode in range of 0.7 to $1.3 \mathbf{~ m m}$ on sensor's performance was investigated in this study through COMSOL simulation. It was found that the modification of the working electrode radius and the gap between the electrodes has the most significant effect on sensor's performance, while modifying the width of the counter electrode (CE) shows no significant effect. Sensors with $0.9 \mathrm{~mm}$ radius or $2.54 \mathrm{~mm}^{2} \mathrm{WE}$ area and $0.5 \mathrm{~mm}$ gap spacing has shown the optimum performance with $0.026 \mathrm{~A} / \mathrm{m}^{2}$ current density which is contributed by $0.044 \mathrm{pF}$ capacitance value. As a conclusion, regardless of the width of counter electrodes, a smaller gap between electrodes and a smaller working area would lead to optimal performance of a screenprinted three-electrode sensor system.
\end{abstract}

Keywords - sensor, screen printing, performance, screenprinted electrode, geometry

\section{INTRODUCTION}

Sensors with high sensitivity, selectivity, and efficiency are becoming increasingly popular in recent years. Recently, it is found that many applications in diverse industries such as environmental, drug and healthcare, food and beverages, pharmaceutical or clinical laboratories, and agricultural used screen-printed sensors with electrochemical systems as main basis or as a whole part [1], [2]. For example, the nicotine sensor created by researchers in [3] is a screen-printed sensor based on electrochemical detection that has been used to quantify nicotine levels in sweats of heavy and light cigarette smokers.

Current electrochemical detectors such as electrophoresis-mass spectrometry (CE-MS), high performance-liquid chromatography (HP-LC) and gas capillary chromatography-mass spectrometry (GC-MS), requires a bulk, high-cost instruments and a complex sample pre-treatment as well [4]-[6]. Screen printed electrodes (SPE) using screen-printing technology, which is based on an electrochemical detection method has emerged as a potential alternative to the complicated chromatography technology because it offers a simpler operation, short analysis time and low-cost approach. Apart from lower costs and bulk manufacturing, the main advantage of this printed electrode sensors is its miniaturized size, which allows the volume of sample required for testing is reduced to as little as a few microliters. This important feature allows the monitoring instrument to be connected to portable instrumentation while reducing its overall size. [2], [7]. However, before it can be used as an effective electrochemical biosensor, standard SPEs must be developed, thus, the optimal configuration of the SPE sensor in terms of sensor structure and geometrical dimensions must be analysed to increase the sensitivity and performance of these electrochemicalbased sensors [8]. Moreover, there is still a lack of discussion about sensor parameter optimization and how it affects electrode electrochemical efficiency[1].

The aim of this research is to develop the best SPE sensor configuration to obtain the optimum performance. The key parameter of the targeted device is sensitivity in terms of high current density and capacitance. This research 
is significant because it explains how and why different geometrical designs of electrodes behave differently in a SPE system, thus paving the way forward for future biosensor development. The device configuration has been designed, and the sensor's performance has been demonstrated using COMSOL software.

\section{DESIGN AND THEORY}

The electrochemical SPE sensor in this work consist of three-electrode system which are working electrode (WE), counter electrode (CE) and reference electrode (RE). Standard SPE is briefly made up of a substrate in which the three electrodes are printed on top of it using the screenprinting technique. The WE serves as the primary electrode to perform the electrochemical reactions, while both RE and $\mathrm{CE}$ are used to complete the electronic circuit [7].

In this simulation work, the materials chosen for WE and $\mathrm{CE}$ is carbon and silver for the RE. Carbon has good flexibility and excellent electrochemical properties while silver is used because it is stable, easy to handle and cheap [9]. Thus, three electrochemistry electrode systems have been completed as depicted in Fig. 1. For specific electrochemical reaction, targeted agent will be immobilised on the working electrode. If the process of immobilization is successful, the attachment of physical nano-molecular on the surface of the carbon electrode will change the impedance and diffusion current [1]. However, this work will focus on simulation of geometrical designs of SPE for optimum performance in terms of current density and capacitance, without considering the electrochemistry part of it.

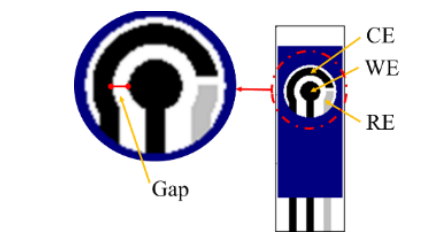

Fig. 1: Screen printed electrode structure

The purpose of the simulations described in this paper is to find the optimal gap size, radius of the WE and width of CE to improve the SPE's efficiency based on the equation (1) $[1]$,

$$
d=\frac{\varepsilon \cdot \mathrm{A}}{C}
$$

where $\mathrm{d}$ is gap between the WE and CE, A is area of working electrode, $\mathrm{C}$ is total capacitance and $\varepsilon$ is permittivity of free space. According to the equation, the capacitance is inversely proportional to the gap spacing between the parallel WE and CE. As a result, reducing the distance between these two electrodes could result in higher capacitance.

In addition to the COMSOL's simulation, the theory of Butler-Volmer equation (2) can be studied to investigate the relationship between current density and total capacitance over the electrode surface area. According to this theory, the capacitance is proportional to the current density, which means that as capacitance increases, the current density and sensitivity increase. Furthermore, since the active geometric area is inversely proportional to current density, reducing the size of geometric area of WE will also increase the current density and the sensor sensitivity [1].

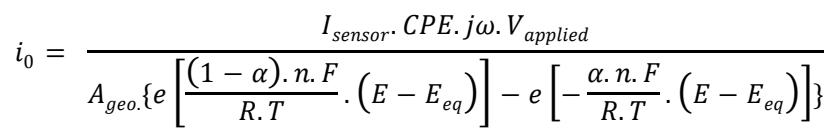

$\mathrm{i}_{0}=$ current density, $\mathrm{A} / \mathrm{m}^{2}$

$\mathrm{A}_{\text {geo }}=$ area of electrode active geometry, $\mathrm{m}^{2}$

$\mathrm{I}_{\text {sensor }}=$ total sensing current, $\mathrm{A}$

$\mathrm{C}_{\text {total }}=\mathrm{CPE}=$ total capacitance, $\mathrm{F}$

$\mathrm{E}=$ electrode potential, $\mathrm{V}$

$\mathrm{V}_{\text {applied }}=$ applied potential, $\mathrm{V}$

$\mathrm{E}_{\text {eq }}=$ equilibrium potential, $\mathrm{V}$

$\mathrm{T}=$ absolute temperature, $\mathrm{K}$

$\mathrm{n}=$ number of electrons involved in reaction

$\mathrm{F}=$ Faraday constant $=96485 \mathrm{C} \mathrm{mol}^{-1}$

$\mathrm{R}=$ universal gas constant $=8.3145 \mathrm{~J} / \mathrm{mol} . \mathrm{K}$

$\alpha=$ symmetry factor

\section{SIMULATION}

In most of product development, it is necessary to solve several variations of a model to find its optimal properties. Instead of manually changing these property values and resolving them each time, the parametric sweep features in COMSOL allows changing the parameter values through a specified range. Using a finite element method with COMSOL Multiphysics software, the effects of gap size, the radius of $\mathrm{WE}$ and the width of $\mathrm{CE}$ were simulated and analyzed. To compare the sensor performance in terms of current density and total capacitance, the gaps between electrodes, radius of WE and the width of CE were varied. Higher current density and total capacitance mean higher magnitude response, so the best combination of electrodes gap, radius of WE and width of CE can be identified. These higher magnitude responses are significant in electrochemical biosensors because they will induce high electro-migration of ions mobility, resulting in higher sensitivity of the sensor [1].

To begin, an electrical AC/DC 3D module was chosen as the simulation model for this analysis. Since it was ideal to measure the current density in conducting devices under conditions where inductive effects are negligible, the physics being used is electric current (ec). Only two electrodes, WE and CE, were used to obtain the ideal gap and radius of WE as shown in Fig. 2. RE is not considered because RE is only used to complete the circuit and maintain the constant potential produced by $\mathrm{CE}$ and $\mathrm{WE}$ [2]. Example of some SPE's with different WE areas is shown in Fig. 3. Table 1 represents the other parameters used in COMSOL simulation.

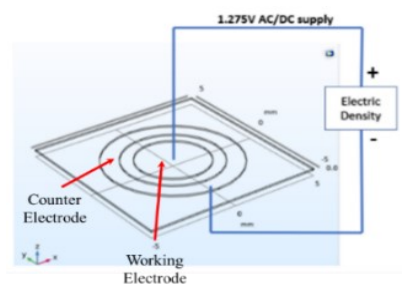

Fig. 2 : Set up of boundary condition for WE and CE using AC/DC module 


\section{ำตำ}

Fig. 3: Designs of SPCE with different radius of WE

A global parameter of the gap size was set to $0.5 \mathrm{~mm}$ to avoid interfering with the measurements of the other electrodes during gap size adjustment. Then, the parametric sweep range is set from $0.5 \mathrm{~mm}$ to $1.6 \mathrm{~mm}$. Apart from the gap dimensions, the same analyses also are performed with defined parameter radius of WE ranging from $0.9 \mathrm{~mm}$ to 2 $\mathrm{mm}$ and width of CE between $0.7 \mathrm{~mm}$ to $1.3 \mathrm{~mm}$. The CE and $\mathrm{WE}$ are given a ground potential and terminal voltage of $1.275 \mathrm{~V}$, and the conducting medium was water. The applied voltage of $1.275 \mathrm{~V}$ was chosen to match with the voltage given to the measurements in future works later. The other parameter such as electrical conductivity and relative permittivity is needed for COMSOL to compute the current density. For mesh setting, the sequence type selected is physics-controlled mesh which is simple, unstructured tetrahedral mesh and the element size chosen is extremely fine to give more accurate results for this simulation. The meshing sequence; size, and free tetrahedral node are auto set by COMSOL, and the nonuniform mesh is automatically produced and customized for the model's physics settings as illustrated in Fig. 4.

Table 1: Parameters used in COMSOL simulation.

\begin{tabular}{|c|l|c|c|}
\hline \multirow{2}{*}{ Parameter } & \multicolumn{3}{|c|}{ COMSOL SIMULATION } \\
\cline { 2 - 4 } & \multicolumn{1}{|c|}{ Definition } & Value & Unit \\
\hline rad_we & Radius of Working Electrode & $\begin{array}{c}0.91 .11 .4 \\
1.72\end{array}$ & $\mathrm{~mm}$ \\
\hline gap & Gap Spacing Between WE and CE & $\begin{array}{c}0.50 .71 .0 \\
1.31 .6\end{array}$ & $\mathrm{~mm}$ \\
\hline width_ce & Width of Counter Electrode & 0.71 .01 .3 & $\mathrm{~mm}$ \\
\hline V1 & Voltage Applied & 1.275 & $\mathrm{~V}$ \\
\hline cond_water & Electrical Conductivity of Water & $5.5 e^{-6}$ & $\mathrm{~S} / \mathrm{m}$ \\
\hline perm_water & Relative Permittivity of Water & 80 & 1 \\
\hline cond_carbon & Electrical Conductivity of Carbon & $3 e^{5}$ & $\mathrm{~S} / \mathrm{m}$ \\
\hline perm_carbon & Relative Permittivity of Carbon & 12 & 1 \\
\hline
\end{tabular}

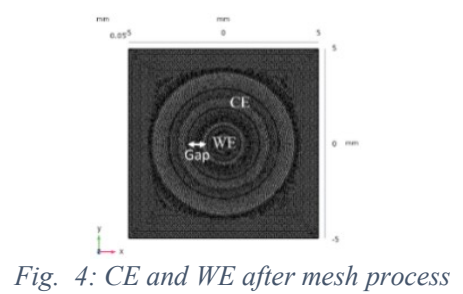

\section{RESUlTS AND DISCUSSION}

\section{A. Current Density}

The magnitude of current density at the edge of WE and $\mathrm{CE}$ was represented by the red line $(\mathrm{a}, \mathrm{b})$ in Fig. 5. With defined gaps ranging from $0.5 \mathrm{~mm}$ to $1.6 \mathrm{~mm}$, and radius of WE range from $0.9 \mathrm{~mm}$ to $2 \mathrm{~mm}$ the analyses were completed.

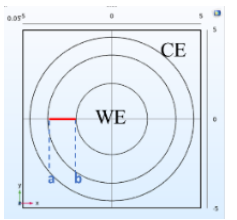

Fig. 5: The red line $(a, b)$ indicates the electrodes gap where $a$ is the edge of $C E$ and $b$ is the edge of WE

According to the simulation results in Fig. 6a, the smallest gap of $0.5 \mathrm{~mm}$ resulting in the highest current density due to the interference electric field of asymmetrical design while the largest gap, $2 \mathrm{~mm}$ resulting the lowest current density. Additionally, reducing the scale of the WE geometric area would increase the current density as shown in Fig. 6b. However, it was found that the changes of width of CE did not really affect the magnitude of current density as shown in Fig. 7, implying that the sensitivity of sensor is unaffected by the width of CE. The main explanation for this is that the main purpose of $\mathrm{CE}$ is only to keep the WE at a constant potential relative to the $\mathrm{RE}$, and thus it has no control on the sensor's efficiency.

In general, when the gap spacing between the electrodes and the radius of the WE were modified, the current density changed. As shown in Fig. 6a and 6b, the smallest gap and radius of WE resulted in the highest current density, therefore a gap of $0.5 \mathrm{~mm}$ and a radius of $0.9 \mathrm{~mm}$ were chosen as the most optimum parameters for producing efficient SPE sensors for electrochemical-based sensors. This simulation of current density agrees with the ButlerVolmer theory described in (2) in Section II. Considering the current density is inversely proportional to active working area (Ageo), thus minimizing the working electrode surface area would result in increased current density.
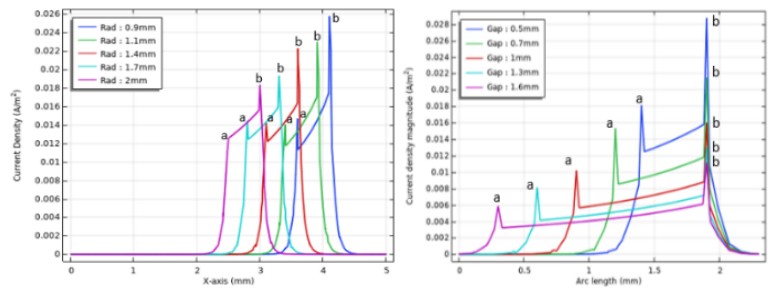

Fig. 6: The current density magnitude of gap: $0.5 \mathrm{~mm}$ (left) and the current density magnitude of radius WE: $0.9 \mathrm{~mm}$ (right)

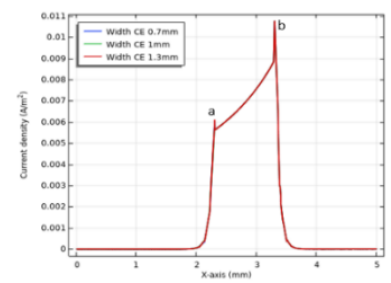

Fig. 7: The current density magnitude with varying width of $C E$

\section{B. Electric Field Magnitude}

The electric field generated by the electrodes is depicted in Fig. 8. It can be seen in the contour graph, regardless the gap between the electrodes, the electric field generated was strong near the WE and gradually decrease towards the edge of CE. In Fig. 9, it is shown that the electric field is greater when the gap spacing between the electrodes is 
smaller. Besides, as the immobilized agent will adhere to the carbon surface of the WE, a higher magnitude of electric field concentrated on the electrode could achieve better sensitivity than a scattered electric field.

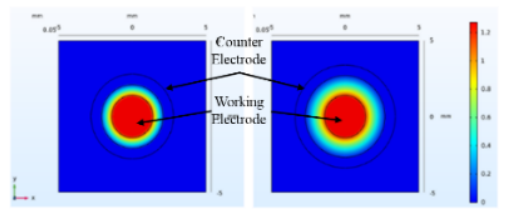

Fig. 8: Electric field distribution on the electrodes surface for $0.7 \mathrm{~mm}$ gap spacing (left) and $1.3 \mathrm{~mm}$ gap spacing (right)

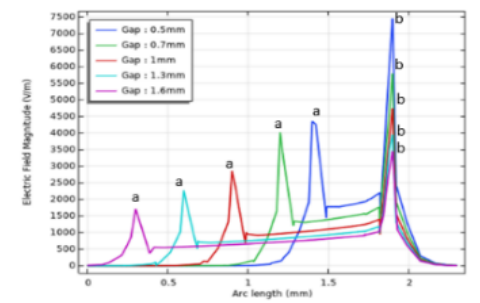

Fig. 9: Electric field magnitude for different gap between WE and CE

\section{Capacitance Value}

The experimental results can be predicted by the COMSOL's simulation, which showed that a smaller gap between the two electrodes would result in higher capacitance and lower impedance value. Smaller spacing, on the other hand, makes the electrodes more sensitive to the activities or any chemical reactions as shown in Fig. 10a and Fig. 10b. As a result, the findings show that the expected outcome from the theoretical analysis on the electrodes gap in equation (1) and the simulation results are in agreement with each other. Both the simulation and theoretical analysis show the same trend, in which capacitance is increasing with smaller gap. In addition, according to equation (2), capacitance (C) is inversely proportional to the gap spacing (d) between the working electrode and the counter electrode, implying that reducing the gap between these two electrodes will increase capacitance and enhance current density in SPE sensors.

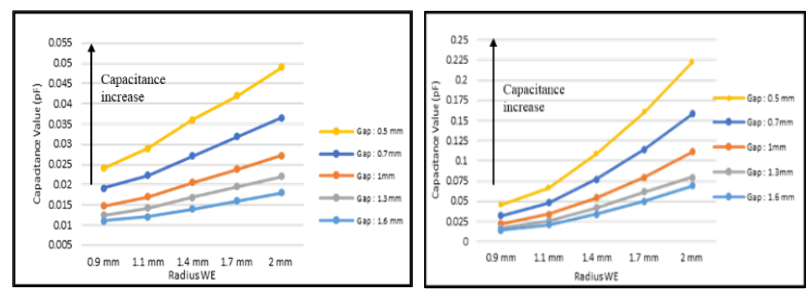

Fig. 10: Simulation of capacitance for gap of $0.5 \mathrm{~mm}$ to $1.6 \mathrm{~mm}$ and radius WE of $0.9 \mathrm{~mm}$ to $2 \mathrm{~mm}$ (left) and theoretical calculation of capacitance based on Butler-Volmer equation for same the dimension (right)

\section{CONCLUSION}

The optimal parameters of electrochemical-based SPE which are widely used for various applications has been successfully simulated by COMSOL Multiphysics software. These evaluations of the effect of geometrical design of SPEs in terms of gap spacing between electrodes, the effect of radius of WE and the effect of width of CE to the performance of the SPE had been discussed and compared with the theoretical analysis. During the simulation, it was observed that modifying the gap between electrodes and the radius of the WE influence the current density value. The maximum peak current density of 0.026 $\mathrm{A} / \mathrm{m}^{2}$ was obtained by combining the smallest electrode gap of $0.5 \mathrm{~mm}$ with the smallest WE radius of $0.9 \mathrm{~mm}$, both of which are contributed by a capacitance value of $0.044 \mathrm{pF}$; these two smallest values is chosen as the optimal parameters of the SPE. The simulations also demonstrate that changing the width of the CE has no influence on the performance of the SPE sensor thus, produce constant value of current density regardless of the width. Future work with these simulations will be focused on fabrication of the sensor and simulation of cyclic voltammetry $(\mathrm{CV})$ to study the electrochemical mechanisms of the electrode using the optimal measurements discuss in this work.

\section{ACKNOWLEDGEMENT}

This project is sponsored by International Islamic University Malaysia under grant project RMCG20-0140014. The authors would like to thank Jabil Circuit Sdn. Bhd. for funding the research materials.

\section{REFERENCES}

A. A. Zainuddin, A. N. Nordin, R. A. Rahim, and W. C. Mak, "Modeling of a novel biosensor with integrated mass and electrochemical sensing capabilities," IECBES 2016 - IEEEEMBS Conf. Biomed. Eng. Sci., pp. 420-425, 2016.

[2] Z. Taleat, A. Khoshroo, and M. Mazloum-Ardakani, "Screenprinted electrodes for biosensing: A review (2008-2013)," Microchim. Acta, vol. 181, no. 9-10, pp. 865-891, 2014.

[3] E. Mehmeti, T. Kilic, C. Laur, and S. Carrara, "Electrochemical determination of nicotine in smokers' sweat," Microchem. J., vol. 158, no. June, 2020.

[4] V. V. Gholap, L. Kosmider, and M. S. Halquist, "A Standardized Approach to Quantitative Analysis of Nicotine in e-Liquids Based on Peak Purity Criteria Using HighPerformance Liquid Chromatography," J. Anal. Methods Chem., vol. 2018, 2018.

[5] A. M. Hossain and S. M. Salehuddin, "Analytical determination of nicotine in tobacco leaves by gas chromatography-mass spectrometry," Arab. J. Chem., vol. 6, no. 3, pp. 275-278, 2013. A. Karthika, P. Karuppasamy, S. Selvarajan, A. Suganthi, and M. Rajarajan, "Electrochemical sensing of nicotine using CuWO4 decorated reduced graphene oxide immobilized glassy carbon electrode," Ultrason. Sonochem., vol. 55, no. December 2018, pp. 196-206, 2019.

[7] A. Hayat and J. L. Marty, "Disposable screen printed electrochemical sensors: Tools for environmental monitoring," Sensors (Switzerland), vol. 14, no. 6, pp. 10432-10453, 2014.

[8] D. E. Garcia, T. H. Chen, F. Wei, and C. M. Ho, "A Parametric Design Study of an Electrochemical Sensor," J. Lab. Autom., vol. 15, no. 3, pp. 179-188, 2010.

[9] K. Yamanaka, M. C. Vestergaard, and E. Tamiya, "Printable electrochemical biosensors: A focus on screen-printed electrodes and their application," Sensors (Switzerland), vol. 16, no. 10, pp. 1-16, 2016. 